\title{
THE X-RAY TRANSFORM ON A GENERAL FAMILY OF CURVES ON FINSLER SURFACES
}

\author{
YERNAT M. ASSYLBEKOV AND NURLAN S. DAIRBEKOV
}

\begin{abstract}
For a compact oriented Finsler surface with smooth boundary, we consider the scalar and vector integral geometry problems over a general family of curves running between boundary points and parametrized by arclength. We impose a natural condition which results in the no conjugate points condition in the case when the curves in question are geodesic lines. Our main theorem generalizes Mukhometov's theorem in several directions.

We also consider these problems on a closed oriented Finsler surface. In this case the integral geometry problems make sense provided that sufficiently many curves in the family are periodic. To this end, we assume that the induced flow on the unit circle bundle of the surface is Anosov. Also, we study the cohomological equation of thermostats without conjugate points.
\end{abstract}

\section{INTRODUCTION}

1.1. Surfaces with boundary. Let $M$ be a compact oriented surface (twodimensional manifold) with boundary. Let $\Gamma$ be a family of regular parametrized curves in $M$ between boundary points such that

(A1) For every interior point $x \in M$ and every $v \in T_{x} M \backslash\{0\}$, there is exactly one curve in $\Gamma$ passing through $x$ in the direction of $v$ (considering the curves obtained by shift of the parameter to be the same curve).

We denote by $\gamma_{x, v}$ such a curve with its parameter shifted so that $\gamma_{x, v}(0)=x$, $\dot{\gamma}_{x, v}(0)=c v(c>0)$. We suppose our family $\Gamma$ to be smooth in the sense that

(A2) The partial map

$$
(x, v, t) \mapsto \gamma_{x, v}(t)
$$

is $C^{\infty}$-smooth.

We assume our family $\Gamma$ to have no conjugate points in the sense that

(A3) The partial map $\exp _{x}^{\Gamma}: T_{x} M \backslash\{0\} \rightarrow M$, defined as

$$
\exp _{x}^{\Gamma}\left(t \dot{\gamma}_{x, v}(0)\right)=\gamma_{x, v}(t), \quad t>0
$$


is a local diffeomorphism for every $x \in M$.

Let $f$ be a smooth function and $\alpha$ be a smooth 1-form on $M$. Define the $X$-ray transform of the function $\psi(x, v)=f(x)+\alpha_{x}(v)$ by

$$
I_{\Gamma} \psi(\gamma)=\int_{\gamma}\left\{f(\gamma(t))+\alpha_{\gamma(t)}(\dot{\gamma}(t))\right\} d t, \quad \gamma \in \Gamma,
$$

meaning the integral over $\gamma$ to stand for the integral over the domain of the parameter. This transform embraces the scalar $(\alpha=0)$ and vector $(f=0)$ X-ray transforms as particular instances, and cannot be decoupled in general, as can be seen, for example, from the case of $\Gamma$ being the family of magnetic geodesics (see [9]). It is clear that $I_{\Gamma}$ has a non-trivial kernel since

$$
I_{\Gamma} d h(\gamma)=\int_{\gamma} d h=0
$$

for all $\gamma \in \Gamma$ and for any $h \in C^{\infty}(M)$ such that $\left.h\right|_{\partial M}=0$. A natural question of integral geometry is whether these are the only elements of the kernel.

Since the integral of a scalar function over a curve respects the parametrization of the curve, we assume $M$ to be furnished with some Finsler metric $F$ and consider the condition:

(A4) All curves in $\Gamma$ are parametrized by arclength with respect to $F$ :

$$
F(\dot{\gamma}(t))=1
$$

for all $t$ and every $\gamma \in \Gamma$.

Our first result is the following:

Theorem 1.1. Let $M$ be a compact oriented surface with boundary, let $F$ be a Finsler metric on $M$, and let $\Gamma$ be a family of curves in $M$ between boundary points satisfying conditions (A1)-(A4). Suppose $\psi(x, v)=f(x)+$ $\alpha_{x}(v)$, where $f$ is a smooth function and $\alpha$ is a smooth 1-form on $M$. Then $I_{\Gamma} \psi(\gamma)=0$ for all $\gamma \in \Gamma$ if and only if $f=0$ and $\alpha=d h$ for some $h \in C^{\infty}(M)$ such that $\left.h\right|_{\partial M}=0$.

In 18, R. G. Mukhometov proved a similar result for a general family of curves on subdomains of the Euclidean plane. In higher dimension, the scalar integral geometry problem for a real analytic family of curves was solved by B. Frigyik, P. Stefanov and G. Uhlmann [10]. It is worth noting that the literature on the question is abandoned in the case when $\Gamma$ is the family of geodesics of a Riemannian metric.

In a purely vectorial case (when $f=0$ ), we may freely reparametrize curves in $\Gamma$ without influencing the X-ray transform. Therefore, we have the following consequence of Theorem 1.1; 
Corollary 1.2. Let $M$ be a compact oriented surface with boundary, let $\Gamma$ be a family of curves in $M$ between boundary points satisfying (A1)-(A3), and let $\alpha$ be a smooth 1 -form on $M$. Then $I_{\Gamma} \alpha(\gamma)=0$ for all $\gamma \in \Gamma$ if and only if $\alpha=d h$ for some $h \in C^{\infty}(M)$ such that $\left.h\right|_{\partial M}=0$.

In [13, S. Holman and P. Stefanov solved the vector integral geometry problem for a real analytic family of curves in any dimension.

1.2. Closed surfaces. A similar problem for closed surfaces is interesting as well. Let $M$ be a closed (i.e., compact and boundaryless) oriented surface, and let $\Gamma$ be a family of regular parametrized curves each of which is defined on the whole real axis.

Assume (A1) and (A2), and let $f$ be a smooth function and $\alpha$ a smooth 1 -form on $M$. Define the $X$-ray transform of the function $\psi(x, v)=f(x)+$ $\alpha_{x}(v)$ by

$$
I_{\Gamma} \psi(\gamma)=\int_{\gamma}\left\{f(\gamma(t))+\alpha_{\gamma(t)}(\dot{\gamma}(t))\right\} d t \quad \text { for periodic } \gamma \in \Gamma,
$$

meaning the integral over $\gamma$ to stand for the integral over the least period.

As before, $I_{\Gamma}$ has a non-trivial kernel since $I_{\Gamma} d h(\gamma)=0$ for all periodic $\gamma \in \Gamma$ and for any $h \in C^{\infty}(M)$. In this setting, the integral geometry problem asks whether these are the only elements in the kernel. Of course, sufficiently many curves in $\Gamma$ must be periodic for this to be true. If $F$ is a Finsler metric on $M$ and (A4) holds, $\Gamma$ defines a flow $\phi_{t}$ on the unit circle bundle by the rule

$$
\phi_{t}(x, v) \mapsto\left(\gamma_{x, v}(t), \dot{\gamma}_{x, v}(t)\right)
$$

Instead of (A3) we require

(A3') The flow $\phi_{t}$ on $S M$ is Anosov.

Recall that the Anosov property means that there is a continuous invariant splitting $T(S M)=\mathbb{R} \mathbf{F} \oplus E^{u} \oplus E^{s}$ (F being the generator of the flow) in such a way that there are constants $C>0$ and $0<\rho<1<\eta$ such that for all $t>0$ we have

$$
\left\|\left.d \phi_{-t}\right|_{E^{u}}\right\| \leq C \eta^{-t} \quad \text { and } \quad\left\|\left.d \phi_{t}\right|_{E^{s}}\right\| \leq C \rho^{t},
$$

where the norms are taken with respect to the Sasaki type Riemannian metric on $S M$ induced by the Finsler metric $F$.

Theorem 1.3. Let $(M, F)$ be a closed oriented Finsler surface, and let $\Gamma$ be a family of curves in $M$ satisfying (A1)-(A2), (A3'), and (A4). Suppose $\psi(x, v)=f(x)+\alpha_{x}(v)$, where $f$ is a smooth function and $\alpha$ is a smooth 1-form on $M$. Then $I_{\Gamma} \psi(\gamma)=0$ for all $\gamma \in \Gamma$ if and only if $f=0$ and $\alpha=d h$ for some $h \in C^{\infty}(M)$. 
In [1], V. Guillemin and D. Kazhdan proved Theorem 1.3 for $F$ a negatively curved Riemannian metric and $\Gamma$ the family of unit-speed geodesics of $F$. A similar result was obtained by G. P. Paternain for magnetic flows in 20]. All these results were based on Fourier analysis. In [8], N. S. Dairbekov and G. P. Paternain proved Theorem 1.3 for the case of the magnetic flow on a Riemannian surface. The same result was proved in [6] by N. S. Dairbekov and G. P. Paternain for thermostats on Riemannian surfaces and in [7] for magnetic flows on Finsler manifolds of any dimension.

1.3. Thermostats. The above-mentioned general families of curves on surfaces are conveniently defined in terms of (generalized) thermostats.

Consider as before a compact oriented surface $M$ and a Finsler metric $F$ on $M$. Let $S M$ be the unit circle bundle of $(M, F)$ and $\pi: S M \rightarrow M$ be the canonical projection, $\pi(x, v)=x$. For any $\lambda \in C^{\infty}(T M \backslash\{0\})$, consider the equation

$$
\frac{D \dot{\gamma}}{d t}=\lambda(\gamma, \dot{\gamma}) i \dot{\gamma}
$$

where $i$ indicates the rotation by $\pi / 2$ according to the orientation of $M$. Every solution of (11) has constant speed, and we restrict ourselves to unitspeed solutions. In this case, it suffices to assume that $\lambda \in C^{\infty}(S M)$. A curve parametrized by arclenth and satisfying (1) will be referred to as $\lambda$ geodesic. We call the triple $(M, F, \lambda)$ a (generalized) thermostat. In case $\lambda$ is (the pullback of) a function on $M$, we have a magnetic system (see, for example, [9]). If $\lambda$ is a 1 -form (regarded as a function on $S M$ ), we have a Gaussian thermostat (see, for example, [6]).

In the case when $M$ has boundary, we assume that the thermostat in question is nontrapping in the sense that every $\lambda$-geodesic has finite exit times both in the positive and negative directions. If $M$ is a closed surface, we assume that that every $\lambda$-geodesic is complete, i.e., defined on the whole real axis. In these cases, we can declare $\Gamma$ to be the family of $\lambda$-geodesics of our thermostat. Then (A1), (A2), and (A4) are obviously satisfied. On the other hand, it is easy to see that the converse is true as well.

Theorem 1.4. If $\Gamma$ is a family of curves on a compact oriented Finsler surface $(M, F)$, satisfying (A1), (A2) and (A4), then it is the family of $\lambda$-geodesics for a suitable $\lambda$.

Proof. Define $\lambda$ as

$$
\lambda(x, v, t)=\left\langle\frac{D \dot{\gamma}_{x, v}(t)}{d t}, i \dot{\gamma}_{x, v}(t)\right\rangle_{\dot{\gamma}_{x, v}(t)},
$$


the inner product on the right-hand side taken with respect to the fundamental tensor in Finsler geometry:

$$
g_{i j}(x, v)=\frac{1}{2}\left[F^{2}\right]_{v^{i} v^{j}}(x, v) .
$$

Condition (A1) implies that the function $\lambda$ does not depend on $t$. Then $\Gamma$ becomes the family of $\lambda$-geodesics of the thermostat $(M, F, \lambda)$.

1.4. Cohomological equation. The cohomological (or kinetic) equation of a flow is simply $\mathbf{F}(u)=\psi$, where $\mathbf{F}$ is the infinitesimal generator of the flow and $u, \psi$ are functions on $S M$. The importance of the cohomological equation in dynamical systems is well known; it arises for example in the study of invariant measures, conjugacy problems, reparametrizations, rigidity questions and inverse problems.

It follows from [12] that under condition (A3') the flow $\phi_{t}$ is transitive and, by the smooth Livčic theorem [16], Theorem 1.3 admits an equivalent restatement as an inverse problem for the cohomological equation. On taking Theorem 1.4 into account, we formulate the corresponding result in terms of thermostats.

Theorem 1.5. Let $(M, F, \lambda)$ be an Anosov thermostat on a closed oriented Finsler surface $M$, and let $\mathbf{F}$ be the infinitesimal generator of the thermostat flow $\phi_{t}$. Suppose $\psi(x, v)=f(x)+\alpha_{x}(v)$, where $f$ is a smooth function and $\alpha$ is a smooth 1-form on $M$. Then the cohomological equation $\mathbf{F}(u)=\psi$ has a solution $u \in C^{\infty}(S M)$ if and only if $f=0$ and the form $\alpha$ is exact.

In case $\psi$ is a scalar function on $M$ (i.e., $\alpha=0$ ), this theorem can be generalized to thermostats without conjugate points. We say that a thermostat has no conjugate points if the family of $\lambda$-geodesics satisfies condition (A3), i.e., if the exponential map

$$
\exp _{x}^{\lambda}(v):=\gamma_{x, v}(|v|), \quad v \neq 0,
$$

is a local diffeomorphism for every $x$. Here $\gamma_{x, v}(t)$ is a unit-speed $\lambda$-geodesic with $\gamma_{x, v}(0)=x$ and $\dot{\gamma}_{x, v}(0)=v /|v|$.

Theorem 1.6. Let $(M, F, \lambda)$ be a thermostat without conjugate points on a closed oriented Finsler surface $M$. Suppose $\psi(x, v)=f(x)$, where $f$ is a smooth function on $M$. Then the cohomological equation $\mathbf{F}(u)=\psi$ has a solution $u \in C^{\infty}(S M)$ if and only if $f=0$.

Note that if a flow is Anosov, then there are no conjugate points (see Subsection 4.1). There are several interesting examples of geodesic flows without conjugate points which are not Anosov and have regions of positive curvature (see [2, 5]). Examples of magnetic flows without conjugate points which are not Anosov are also given in [5]. 
Theorem 1.1, too, is proved by a reduction to an inverse problem for the cohomological equation on a thermostat. The reduction follows the arguments in [4] and is based on the previous observations by V. A. Sharafutdinov in 22 .

In all the cases, the cohomological equation is analyzed by means of Pestov type identities that we derive in each case.

1.5. Structure of the paper. The organization of the paper is as follows. In Section 2 we combine certain preliminaries concerning thermostats on Finsler surfaces, as well as derive differential and integral Pestov type identities. Section 3 is devoted to the proof of Theorem 1.1. Section 4 contains the proof of Theorem 1.3. Here we also give sufficient conditions in terms of the Finsler metric and thermostat data for a thermostat flow to be Anosov (see Theorem 4.5). The closing Section 5 contains the proof of Theorem 1.6.

\section{Pestov identity}

2.1. Canonical coframing. By [3, Chapter 4] for a given Finsler surface $(M, F)$ it is possible to define a canonical coframing $\left(\omega_{1}, \omega_{2}, \omega_{3}\right)$ on $S M$ that satisfies the following structural equations:

$$
\begin{aligned}
& d \omega_{1}=-\omega_{2} \wedge \omega_{3}, \\
& d \omega_{2}=-\omega_{3} \wedge\left(\omega_{1}-I \omega_{2}\right), \\
& d \omega_{3}=-\left(K \omega_{1}-J \omega_{3}\right) \wedge \omega_{2} .
\end{aligned}
$$

where $I, K$ and $J$ are smooth functions on $S M$. The function $I$ is called the main scalar of the structure. When the Finsler structure is Riemannian, $K$ is the Gaussian curvature.

Consider the vector fields $(X, H, V)$ dual to $\left(\omega_{1}, \omega_{2}, \omega_{3}\right)$. As a consequence of (3) they satisfy the commutation relations

$$
\begin{aligned}
& {[V, X]=H,} \\
& {[H, V]=X+I H+J V,} \\
& {[X, H]=K V .}
\end{aligned}
$$

Let $\lambda$ be a smooth function on $S M$ and let $\mathbf{F}=X+\lambda V$ be the generating vector field of the thermostat $(M, F, \lambda)$. From (6) we obtain:

$$
\begin{aligned}
& {[V, \mathbf{F}]=H+V(\lambda) V,} \\
& {[H, V]=\mathbf{F}+I H+(J-\lambda) V,} \\
& {[\mathbf{F}, H]=\left\{K-H(\lambda)-\lambda J+\lambda^{2}\right\} V-\lambda \mathbf{F}-\lambda I H .}
\end{aligned}
$$




\subsection{Pestov identity.}

Lemma 2.1 (Pestov identity). For every smooth function $u: S M \rightarrow \mathbb{R}$ we have

$$
\begin{array}{r}
2 H u \cdot V \mathbf{F} u=(\mathbf{F} u)^{2}+(H u)^{2}-\left(K-H(\lambda)-\lambda J+\lambda^{2}\right)(V u)^{2} \\
+\mathbf{F}(H u \cdot V u)-H(V u \cdot \mathbf{F} u)+V(H u \cdot \mathbf{F} u) \\
+\mathbf{F} u \cdot(I H u+J V u)+H u \cdot V u \cdot(\lambda I+V(\lambda)) .
\end{array}
$$

Proof. Using the commutation formulas, we deduce:

$$
\begin{aligned}
2 H u \cdot V \mathbf{F} u-V( & H u \cdot \mathbf{F} u)=H u \cdot V \mathbf{F} u-V H u \cdot \mathbf{F} u \\
= & H u \cdot(\mathbf{F} V u+[V, \mathbf{F}] u)-\mathbf{F} u \cdot(H V u+[V, H] u) \\
= & H u \cdot(\mathbf{F} V u+H u+V(\lambda) V u) \\
& -\mathbf{F} u \cdot(H V u-\mathbf{F} u-I H u-(J-\lambda) V u) \\
= & (H u)^{2}+(\mathbf{F} u)^{2}+H u \cdot \mathbf{F} V u-H V u \cdot \mathbf{F} u+I \mathbf{F} u \cdot H u \\
& +(J-\lambda) \mathbf{F} u \cdot V u+V(\lambda) H u \cdot V u \\
= & (H u)^{2}+(\mathbf{F} u)^{2}+\mathbf{F}(H u \cdot V u)-H(V u \cdot \mathbf{F} u)-[\mathbf{F}, H] u \cdot V u \\
& +I \mathbf{F} u \cdot H u+(J-\lambda) \mathbf{F} u \cdot V u+V(\lambda) H u \cdot V u \\
= & (H u)^{2}+(\mathbf{F} u)^{2}+\mathbf{F}(H u \cdot V u)-H(V u \cdot \mathbf{F} u) \\
& +\left(-\left(K-H(\lambda)-\lambda J+\lambda^{2}\right) V u+\lambda \mathbf{F} u+\lambda I H u\right) \cdot V u \\
& +I \mathbf{F} u \cdot H u+(J-\lambda) \mathbf{F} u \cdot V u+V(\lambda) H u \cdot V u \\
= & (H u)^{2}+(\mathbf{F} u)^{2}+\mathbf{F}(H u \cdot V u)-H(V u \cdot \mathbf{F} u) \\
& -\left(K-H(\lambda)-\lambda J+\lambda^{2}\right)(V u)^{2}+I \mathbf{F} u \cdot H u+J \mathbf{F} u \cdot V u \\
& +(\lambda I+V(\lambda)) H u \cdot V u
\end{aligned}
$$

which is equivalent to the Pestov identity.

For $G$ a vector field and $\Theta$ a differential form, Cartan's formula for the Lie derivative reads:

$$
\mathcal{L}_{G} \Theta=d\left(i_{G} \Theta\right)+i_{G} d \Theta .
$$

Now let $\Theta:=\omega_{1} \wedge \omega_{2} \wedge \omega_{3}$. This volume form gives rise to the Liouville measure $d \mu$ of $S M$.

Lemma 2.2. We have:

$$
\begin{aligned}
& \mathcal{L}_{\mathbf{F}} \Theta=(\lambda I+V(\lambda)) \Theta \\
& \mathcal{L}_{H} \Theta=-J \Theta \\
& \mathcal{L}_{V} \Theta=I \Theta
\end{aligned}
$$


Proof. Using equations (35)

$$
\mathcal{L}_{X} \Theta=d\left(i_{X} \Theta\right)=d\left(\omega_{2} \wedge \omega_{3}\right)=d \omega_{2} \wedge \omega_{3}-\omega_{2} \wedge d \omega_{3}=0
$$

Since $\mathbf{F}=X+\lambda V$, we get

$$
\mathcal{L}_{\mathbf{F}} \Theta=\mathcal{L}_{X} \Theta+\mathcal{L}_{\lambda V} \Theta=d\left(i_{\lambda V} \Theta\right)=-d\left(\lambda \omega_{2} \wedge \omega_{1}\right)=(\lambda I+V(\lambda)) \Theta
$$

Similarly, $\mathcal{L}_{H} \Theta=-J \Theta, \mathcal{L}_{V} \Theta=I \Theta$.

2.3. Pestov integral identity. Integrate the Pestov identity over $S M$ against the Liouville measure $d \mu$ by making use of the Stokes Theorem and (8, 10) to get

$$
\begin{aligned}
\int_{S M} 2 H u \cdot V \mathbf{F} u d \mu & =\int_{S M}(\mathbf{F} u)^{2} d \mu+\int_{S M}(H u)^{2} d \mu \\
& -\int_{S M}\left\{K-H(\lambda)-\lambda J+\lambda^{2}\right\}(V u)^{2} d \mu \\
& +\int_{\partial(S M)}\left\{(H u \cdot V u) i_{\mathbf{F}} \Theta+(\mathbf{F} u \cdot H u) i_{V} \Theta-(\mathbf{F} u \cdot V u) i_{H} \Theta\right\} .
\end{aligned}
$$

Since $i_{V} \Theta=\omega_{1} \wedge \omega_{2}$ vanishes when restricted to $\partial(S M)$, we have

$$
\int_{\partial(S M)}(\mathbf{F} u \cdot H u) i_{V} \Theta=0 .
$$

So we get

$$
\begin{aligned}
\int_{S M} 2 H u \cdot V \mathbf{F} u d \mu & =\int_{S M}(\mathbf{F} u)^{2} d \mu+\int_{S M}(H u)^{2} d \mu \\
& -\int_{S M}\left\{K-H(\lambda)-\lambda J+\lambda^{2}\right\}(V u)^{2} d \mu \\
& +\int_{\partial(S M)}\left\{(H u \cdot V u) i_{\mathbf{F}} \Theta-(\mathbf{F} u \cdot V u) i_{H} \Theta\right\} .
\end{aligned}
$$

By commutation relations, we have

$$
\mathbf{F} V u=V \mathbf{F} u-H u-V(\lambda) V u .
$$


Therefore,

$$
\begin{aligned}
(\mathbf{F} V u)^{2} & =(V \mathbf{F} u)^{2}+(H u)^{2}+(V(\lambda) V u)^{2}-2 V \mathbf{F} u \cdot H u+2 H u \cdot V(\lambda) V u \\
& -2 V \mathbf{F} u \cdot V(\lambda) V u \\
& =(V \mathbf{F} u)^{2}+(H u)^{2}+(V(\lambda) V u)^{2}-2 V \mathbf{F} u \cdot H u+2 H u \cdot V(\lambda) V u \\
& -2 V \mathbf{F} u \cdot V(\lambda) V u+2 V(\lambda) \mathbf{F} V u \cdot V u-2 V(\lambda) \mathbf{F} V u \cdot V u \\
& =(V \mathbf{F} u)^{2}+(H u)^{2}+(V(\lambda) V u)^{2}-2 V \mathbf{F} u \cdot H u+2 H u \cdot V(\lambda) V u \\
& -2 V(\lambda)[V, \mathbf{F}] V u-2 V(\lambda) \mathbf{F} V u \cdot V u \\
& =(V \mathbf{F} u)^{2}+(H u)^{2}-(V(\lambda) V u)^{2}-2 V \mathbf{F} u \cdot H u-2 V(\lambda) \mathbf{F} V u \cdot V u .
\end{aligned}
$$

Since

$$
-2 V(\lambda) \mathbf{F} V u \cdot V u=-\mathbf{F}\left(V(\lambda)(V u)^{2}\right)+(V u)^{2} \mathbf{F} V(\lambda)
$$

we obtain:

$$
\begin{aligned}
(\mathbf{F} V u)^{2} & =(V \mathbf{F} u)^{2}+(H u)^{2}-(V(\lambda) V u)^{2}-2 V \mathbf{F} u \cdot H u \\
& -\mathbf{F}\left(V(\lambda)(V u)^{2}\right)+(V u)^{2} \mathbf{F} V(\lambda) .
\end{aligned}
$$

Integrating it over $S M$ we get

$$
\begin{aligned}
\int_{S M} 2 H u \cdot V \mathbf{F} u d \mu & =\int_{S M}(V \mathbf{F} u)^{2} d \mu+\int_{S M}(H u)^{2} d \mu-\int_{S M}(\mathbf{F} V u)^{2} d \mu \\
& +\int_{S M}\{\mathbf{F} V(\lambda)+\lambda I V(\lambda)\}(V u)^{2} d \mu \\
& -\int_{\partial(S M)} V(\lambda)(V u)^{2} i_{\mathbf{F}} \Theta
\end{aligned}
$$

since by the Stokes Theorem and (8)

$$
\begin{aligned}
-\int_{S M} \mathbf{F}\left(V(\lambda)(V u)^{2}\right) \Theta= & \int_{S M} \lambda I V(\lambda)(V u)^{2} \Theta+\int_{S M}(V(\lambda))^{2}(V u)^{2} \Theta \\
& -\int_{\partial(S M)} V(\lambda)(V u)^{2} i_{\mathbf{F}} \Theta .
\end{aligned}
$$

Combining (11) and (13), we come to the final integral identity:

Theorem 2.3 (Pestov integral identity).

$$
\begin{aligned}
\int_{S M}(\mathbf{F} V u)^{2} d \mu-\int_{S M} \mathbb{K}(V u)^{2} d \mu+ & \int_{\partial(S M)} \omega(u) \\
& =\int_{S M}(V \mathbf{F} u)^{2} d \mu-\int_{S M}(\mathbf{F} u)^{2} d \mu
\end{aligned}
$$


where

$$
\begin{aligned}
& \omega(u):=\left\{(H u \cdot V u)+V(\lambda)(V u)^{2}\right\} i_{\mathbf{F}} \Theta-(\mathbf{F} u \cdot V u) i_{H} \Theta, \\
\text { and } \mathbb{K}:= & K-H(\lambda)-\lambda J+\lambda^{2}+\mathbf{F} V(\lambda)+\lambda I V(\lambda) .
\end{aligned}
$$

\section{Proof of Theorem 1.1}

As mentioned, Theorem 1.1 is proved by reduction to the cohomological equation, followed by the analysis of the latter by means of Pestov type identities. The reduction is performed in Subsections 3.1 and 3.2 and is based on observations in [22], also used in [4], which we formulate below in Theorem 3.1 and Proposition 3.3. Subsection 3.3 is devoted to adaptation of the Pestov integral identity (Theorem 2.3) to the case under study. In Subsection 3.4 we derive one more integral identity which we use to prove the main result. The proof of Theorem 1.1 is completed in Subsection 3.5.

3.1. Preparation. By Theorem 1.4 we can assume that

$$
\int_{\gamma} \psi(\gamma, \dot{\gamma}) d t=0
$$

for all $\lambda$-geodesics $\gamma$ with endpoints on $\partial M$. The aim of this section is to prove the next theorem.

Theorem 3.1. Let $(M, F, \lambda)$ be a nontrapping thermostat without conjugate points. Suppose $\psi(x, v)$ is a smooth function on $S M$ and (15) holds for every $\lambda$-geodesic $\gamma$ with endpoints on $\partial M$, then

$$
\left.\psi\right|_{S(\partial M)}=0 .
$$

Proof. First of all, we extend $\psi$ to a positively homogeneous function of degree zero on $T M \backslash\{0\}$.

Fix $x \in \partial M$ and $v \in S_{x}(\partial M)$. Let $n \in S_{x} M$ be an inward unit vector at $x$. For $\varepsilon>0$ put $v_{\varepsilon}=v+\varepsilon n$ and consider the $\lambda$-geodesic $\gamma_{\varepsilon}=\gamma_{x, v_{\varepsilon}}$. Let $\tau_{\varepsilon}$ is the first time at which $\gamma_{\varepsilon}$ meets the boundary, $\gamma_{\varepsilon}\left(\tau_{\varepsilon}\right) \in \partial M$. So $\gamma_{\varepsilon}:\left[0, \tau_{\varepsilon}\right] \rightarrow M, \gamma_{\varepsilon}(0) \in \partial M, y_{\varepsilon}:=\gamma_{\varepsilon}\left(\tau_{\varepsilon}\right) \in \partial M$, and $\gamma_{\varepsilon}(t) \in M^{\text {int }}$ for $0<t<\tau_{\varepsilon}$. We separately consider two possible cases. First, there is a sequence $0<\varepsilon_{k} \rightarrow 0$ such that $\tau_{\varepsilon_{k}} \rightarrow 0$ as $k \rightarrow \infty$. Second, there is $\tau_{0}>0$ such that $\tau_{\varepsilon} \geq \tau_{0}>0$ for all $0<\varepsilon \leq \varepsilon_{0}$. In the first case, assume (16) fails; for definiteness,

$$
\psi(x, v)>0 .
$$

For $k$ large enough, the points $\left(\gamma_{\varepsilon_{k}}(t), \dot{\gamma}_{\varepsilon_{k}}(t)\right)$ belong to any prescribed neighbourhood of $(x, v)$ for all $t \in\left[0, \tau_{\varepsilon_{k}}\right]$. Therefore, the latter inequality implies that the integrand in

$$
\int_{0}^{\tau_{\varepsilon_{k}}} \psi\left(\gamma_{\varepsilon_{k}}(t), \dot{\gamma}_{\varepsilon_{k}}(t)\right) d t
$$


is strictly positive on $\left(0, \tau_{\varepsilon_{k}}\right)$. Hence, this integral is strictly positive, which contradicts the hypothesis of the theorem.

Now, we consider the second case. Fix $\varepsilon \in\left(0, \varepsilon_{0}\right)$ and let $s \mapsto x_{s} \in \partial M$ $(0 \leq s<\delta)$ be a parametrization of $\partial M$ near $x$ such that $x_{0}=x$ and $\left.\frac{d x_{s}}{d s}\right|_{s=0}=v$.

We first assume that $\gamma_{\varepsilon}$ meets $\partial M$ at $y_{\varepsilon}=\gamma_{\varepsilon}\left(\tau_{\varepsilon}\right)$ transversally. Then for $s$ small enough there is a unique $\lambda$-geodesic $\gamma_{\varepsilon, s}$ from $x_{s}$ to $y_{\varepsilon}$ in $M$. We choose a parametrization of $\gamma_{\varepsilon, s}$ so as to have $\gamma_{\varepsilon, s}:\left[-\tau_{\varepsilon, s}, 0\right] \rightarrow M, \gamma_{\varepsilon, s}\left(-\tau_{\varepsilon, s}\right)=x_{s}$, $\gamma_{\varepsilon, s}(0)=y_{\varepsilon}$. Moreover, $\tau_{\varepsilon, s}$ depends smoothly on $s$ and $\gamma_{\varepsilon, s}(t)$ depends smoothly on $(s, t)$. Henceforth we accordingly shift a parameter on $\gamma_{\varepsilon}$ so that $\gamma_{\varepsilon}=\gamma_{\varepsilon, 0}$.

By hypothesis,

$$
\int_{-\tau_{\varepsilon, s}}^{0} \psi\left(\gamma_{\varepsilon, s}(t), \dot{\gamma}_{\varepsilon, s}(t)\right) d t=0
$$

Taking the derivative with respect to $s$ at $s=0$, we get

$$
\begin{aligned}
\left.\psi\left(x, v_{\varepsilon} /\left|v_{\varepsilon}\right|\right) \frac{d \tau_{\varepsilon, s}}{d s}\right|_{s=0}+\int_{-\tau_{\varepsilon}}^{0}\{ & \frac{\partial \psi}{\partial x^{k}}\left(\gamma_{\varepsilon}(t), \dot{\gamma}_{\varepsilon}(t)\right) J_{\varepsilon}^{k}(t) \\
& \left.+\frac{\partial \psi}{\partial v^{k}}\left(\gamma_{\varepsilon}(t), \dot{\gamma}_{\varepsilon}(t)\right) \frac{D J_{\varepsilon}^{k}(t)}{d t}\right\} d t=0,
\end{aligned}
$$

where $J_{\varepsilon}(t)=\left.\frac{\partial \gamma_{\varepsilon, s}(t)}{\partial s}\right|_{s=0}$ is the variation (Jacobi) field along $\gamma_{\varepsilon}$.

Differentiating the identity $\gamma_{\varepsilon, s}\left(-\tau_{\varepsilon, s}\right)=x_{s}$ with respect to $s$, at $s=0$ we get

On putting

$$
J_{\varepsilon}\left(-\tau_{\varepsilon}\right)-\left.\dot{\gamma}_{\varepsilon}\left(-\tau_{\varepsilon}\right) \frac{d \tau_{\varepsilon, s}}{d s}\right|_{s=0}=v
$$

$$
A_{\varepsilon}=-\left.\frac{d \tau_{\varepsilon, s}}{d s}\right|_{s=0}
$$

and using the equality $\dot{\gamma}_{\varepsilon}\left(-\tau_{\varepsilon}\right)=(v+\varepsilon n) /|v+\varepsilon n|=v+O(\varepsilon)$, we get

$$
J_{\varepsilon}\left(-\tau_{\varepsilon}\right)=v-A_{\varepsilon} \dot{\gamma}_{\varepsilon}\left(-\tau_{\varepsilon}\right)=\left(1-A_{\varepsilon}\right) \dot{\gamma}_{\varepsilon}\left(-\tau_{\varepsilon}\right)+O(\varepsilon) .
$$

Hence, $J_{\varepsilon}$ has the following boundary conditions:

$$
J_{\varepsilon}\left(-\tau_{\varepsilon}\right)=\left(1-A_{\varepsilon}\right) \dot{\gamma}_{\varepsilon}\left(-\tau_{\varepsilon}\right)+O(\varepsilon), \quad J_{\varepsilon}(0)=0 .
$$

As soon as a Jacobi field depends linearly on boundary conditions, from (18) we deduce

$$
J_{\varepsilon}(t)=-\frac{1-A_{\varepsilon}}{\tau_{\varepsilon}} t \dot{\gamma}_{\varepsilon}(t)+\tilde{J}_{\varepsilon}(t)
$$

where $\tilde{J}_{\varepsilon}$ is a Jacobi field along $\gamma_{\varepsilon}$ with boundary conditions

$$
\tilde{J}_{\varepsilon}\left(-\tau_{\varepsilon}\right)=O(\varepsilon), \quad \tilde{J}_{\varepsilon}(0)=0 .
$$


Using these conditions together with the Jacobi equation, which we do not derive here since it can be done similarly as in [7, we conclude that

$$
\tilde{J}_{\varepsilon}=O(\varepsilon)
$$

in an appropriate $C^{1}$-norm.

To evaluate $A_{\varepsilon}$, put $c_{\varepsilon}(s, t)=\gamma_{\varepsilon, s}\left(\left(\tau_{\varepsilon, s} / \tau_{\varepsilon}\right) t\right)$. Then $\tau_{\varepsilon, s}$ is the length of the curve $c_{\varepsilon}(s, \cdot):\left[-\tau_{\varepsilon}, 0\right] \rightarrow M$ and the variation field of $c_{\varepsilon}(s, t)$ is

$V(t)=\left.\frac{\partial c_{\varepsilon}(s, t)}{\partial s}\right|_{s=0}=\left.\frac{\partial \gamma_{\varepsilon, s}\left(\left(\tau_{\varepsilon, s} / \tau_{\varepsilon}\right) t\right)}{\partial s}\right|_{s=0}=J_{\varepsilon}(t)-\frac{A_{\varepsilon}}{\tau_{\varepsilon}} t \dot{\gamma}_{\varepsilon}(t)=-\frac{t \dot{\gamma}_{\varepsilon}(t)}{\tau_{\varepsilon}}+O(\varepsilon)$.

The first variation formula for length, together with (19), now gives

$$
\begin{array}{r}
\left.\frac{d \tau_{\varepsilon, s}}{d s}\right|_{s=0}=-\int_{-\tau_{\varepsilon}}^{0}\left\langle V(t), \frac{D \dot{\gamma}_{\varepsilon}}{d t}\right\rangle_{\dot{\gamma}_{\varepsilon}(t)} d t-\left\langle V\left(-\tau_{\varepsilon}\right), \dot{\gamma}_{\varepsilon}\left(-\tau_{\varepsilon}\right)\right\rangle_{\dot{\gamma}_{\varepsilon}\left(-\tau_{\varepsilon}\right)} \\
=-\int_{-\tau_{\varepsilon}}^{0}\left\langle-\frac{t \dot{\gamma}_{\varepsilon}(t)}{\tau_{\varepsilon}}, \frac{D \dot{\gamma}_{\varepsilon}}{d t}\right\rangle_{\dot{\gamma}_{\varepsilon}(t)} d t-\left\langle\dot{\gamma}_{\varepsilon}\left(-\tau_{\varepsilon}\right), \dot{\gamma}_{\varepsilon}\left(-\tau_{\varepsilon}\right)\right\rangle_{\dot{\gamma}_{\varepsilon}\left(-\tau_{\varepsilon}\right)}+O(\varepsilon) \\
=-1+O(\varepsilon) .
\end{array}
$$

Hence $A_{\varepsilon}=1+O(\varepsilon)$. From (19) and (21) we then get: $J_{\varepsilon}=O(\varepsilon)$ in an appropriate $C^{1}$-norm. Therefore, using (17) we conclude that

$$
\psi(x, v)=\psi\left(x, v_{\varepsilon} /\left|v_{\varepsilon}\right|\right)+O(\varepsilon)=O(\varepsilon) .
$$

We recall that the above argument was carried out under the assumption that $\gamma_{\varepsilon}$ intersects $\partial M$ transversally at the point $y_{\varepsilon}=\gamma_{\varepsilon}\left(\tau_{\varepsilon}\right)$.

To get rid of this assumption, we invoke the following:

Proposition 3.2. [21, Theorem 3.7, Ch. IX] Given a plane set $R$, let $P$ be a subset of $R$ at every point of which the set $R$ has an extreme tangent parallel to a fixed straight line $D$. Then the orthogonal projection of $P$ on the line at right angles to $D$ is of linear measure zero.

Applying this proposition in polar coordinates related to the exponential map (2) at $x$, we conclude that $\gamma_{\varepsilon}$ meets $\partial M$ transversally at $y_{\varepsilon}$ for almost every $\varepsilon$. Hence, (22) holds for all $\varepsilon$, which implies the claim of the theorem.

3.2. Reduction to the kinetic equation. If $\psi(x, v)=f(x)+\alpha_{x}(v)$ satisfies the conditions of Theorem 1.1, we know from Theorem 3.1 that $\psi(x, v)=$ 0 for $(x, v) \in S(\partial M)$. As soon as $\psi(x,-v)=0$ too, we have $f(x)=0$ and $\alpha_{x}(v)=0$ for $x \in \partial M$ and $v \in T_{x}(\partial M)$. The following obvious proposition can also be regarded as an easy consequence of [22, Lemma 2.2]. 
Proposition 3.3. Let $g$ be a Riemannian metric on $M$ and let $n$ be the inward unit normal to $\partial M$ in $M$. If $\theta$ is a smooth function on $\partial M$, then there is $h \in C^{\infty}(M)$ such that $\left.h\right|_{\partial M}=0$ and $\left.\frac{\partial h}{\partial n}\right|_{\partial M}=\theta$.

Considering any Riemannian metric $g$ on $M$ and taking $\theta=\alpha(n)$, it follows that the function $\tilde{\psi}=\psi-d h$ has the following property: the equality

$$
\tilde{\psi}(x, v)=0
$$

holds for all $x \in \partial M$ and $v \in T_{x} M$.

Now we reduce the proof of Theorem 1.1 to an inverse problem for a kinetic equation. In view of (16) and (23), we may henceforth assume that the function $\psi$ itself vanishes on the boundary:

$$
\left.\psi\right|_{\partial(S M)}=0 .
$$

Further, without loss of generality, we assume that $M$ is a subset of a closed smooth surface $U$. We extend $F$ to a Finsler metric on $U$ and extend $\lambda$ to a smooth function on $S U$, thus obtaining a thermostat $(U, F, \lambda)$. We extend $\psi$ from $S M$ to $S U$ by zero, preserving the notation. The boundary condition (24) guarantees that the so-obtained function $\psi$ is continuous on $U$ and belongs to the Sobolev space $H^{1}(S U)$ of square-integrable functions with square-integrable first-order derivatives.

Since $(M, F, \lambda)$ is nontrapping, there is no complete $\lambda$-geodesic which would be contained entirely in $M$. Therefore, for any $(x, v) \in S M$ there is a number $\tau(x, v)$ such that $\gamma_{x, v}(\tau(x, v)) \notin M$. We define a function $u: S U \rightarrow \mathbb{R}$ to be

$$
u(x, v)=\int_{0}^{\tau(x, v)} \psi\left(\phi_{t}(x, v)\right) d t .
$$

Note that the value of $u(x, v)$ is independent of the choice of $\tau(x, v)$. This follows from (15) and the fact that $\psi$ vanishes on $S U \backslash S M^{\text {int }}$.

Call a point $(x, v) \in S M$ regular if the $\lambda$-geodesic $\gamma_{x, v}$ intersects $\partial M$ transversally from either side, and if the open segment of $\gamma_{x, v}$ between the basepoint $x$ and the point of intersection lies entirely in $M^{\text {int }}$. We denote by $R M \subset S M$ the set of all regular points. It is clear that $R M$ is open in $S M$ and has full measure in $S M$.

Lemma 3.4. The function $u: S U \rightarrow \mathbb{R}$ has the following properties:

(i) $\left.u\right|_{S(U \backslash M)}=0$,

(ii) $u \in H^{1}(S U) \cap C(S U) \cap C^{\infty}(R M)$,

(iii) $u$ is $C^{1}$ smooth along the orbits of the thermostat flow $\phi$ and satisfies the following kinetic equation on $S U$ :

$$
\mathbf{F} u(x, v)=-\psi(x, v) .
$$


Proof. Statement (i) is a direct consequence of (15) and (25).

To prove (ii) take any point $(x, v) \in S U$. Since $\gamma_{x, v}(\tau(x, v)) \notin M$, we can choose a small one-dimensional subspace $\Phi$ in $U$ transversally intersecting $\gamma_{x, v}$ at the point $\gamma_{x, v}(\tau(x, v))$ and disjoint from M. Then there is a neighbourhood of $(x, v)$ in $S U$ such that for every $\left(x^{\prime}, v^{\prime}\right)$ in this neighbourhood the $\lambda$-geodesic $\gamma_{x^{\prime}, v^{\prime}}$ will hit $\Phi$ at the time $\tilde{\tau}\left(x^{\prime}, v^{\prime}\right)$ smoothly depending on $\left(x^{\prime}, v^{\prime}\right)$ and such that $\tilde{\tau}(x, v)=\tau(x, v)$. For these $\left(x^{\prime}, v^{\prime}\right)$ we can therefore take $\tilde{\tau}\left(x^{\prime}, v^{\prime}\right)$ as $\tau\left(x^{\prime}, v^{\prime}\right)$ while defining $u$ by (25). So locally the lower limit of integration in (25) can be chosen to be a smooth function. Since $\psi$ is continuous and lies in the Sobolev space $H^{1}(S U)$, this observation allows us to prove routinely that $u$ is continuous and belongs to $H^{1}(S U)$.

If $(x, v) \in R M$, then there is a neighbourhood of $(x, v)$ in $S U$ such that for all $\left(x^{\prime}, v^{\prime}\right)$ in this neighbourhood the $\lambda$-geodesics $\gamma_{x^{\prime}, v^{\prime}}$ intersect $\partial M$ transversally from the same side as $\gamma_{x, v}$ so that the interior of the segment between $x^{\prime}$ and the point of intersection lies in $M^{\text {int }}$ for each of these $\lambda$-geodesics. Moreover, the parameter values $\tau\left(x^{\prime}, v^{\prime}\right)$ of the intersection points are smooth functions of $\left(x^{\prime}, v^{\prime}\right)$ in this neighbourhood. Since $\psi$ is smooth in $S M$, we conclude that $u$ is smooth in the chosen neighbourhood of $(x, v)$ and therefore smooth on $R M$.

Finally, we give the proof of (iii). For $\varphi \in C^{\infty}(S M)$ and $(x, v) \in S M$ we have

$$
\mathbf{F} \varphi(x, v)=\left.\frac{d}{d t} \varphi\left(\phi_{t}(x, v)\right)\right|_{t=0} .
$$

By way of approximation, it is easy to see that (27) works equally well for the functions $\varphi \in H^{1}(S U) \cap C(S U)$. To apply it to our function $u$, take $(x, v) \in S M$. Then $\gamma_{\phi_{s}(x, v)}(t)=\gamma_{x, v}(t+s)$ and we can take $\tau\left(\phi_{s}(x, v)\right)=$ $\tau(x, v)-s$. By (25)

$$
u\left(\phi_{s}(x, v)\right)=\int_{0}^{\tau\left(\phi_{s}(x, v)\right)} \psi\left(\phi_{t+s}(x, v)\right) d t=\int_{s}^{\tau(x, v)} \psi\left(\phi_{t}(x, v)\right) d t .
$$

Taking the derivative at $s=0$ gives (26) and finishes the proof of the lemma.

3.3. Pestov integral identity. Let $D$ be a compact oriented two-dimensional submanifold of $U$ with boundary $\partial D$ and $u: S D \rightarrow \mathbb{R}$ be a smooth function such that $\left.u\right|_{\partial(S D)}=0$. By Theorem 2.3 we have

$$
\int_{S D}(\mathbf{F} V u)^{2} d \mu-\int_{S D} \mathbb{K}(V u)^{2} d \mu=\int_{S D}(V \mathbf{F} u)^{2} d \mu-\int_{S D}(\mathbf{F} u)^{2} d \mu .
$$

Lemma 3.5. Let $D \subset U$ be a surface with boundary $\partial D$. Let a function $u: S D \rightarrow \mathbb{R}$ be such that $u \in H^{1}(S D), \mathbf{F} u \in H^{1}(S D), u$ is smooth in some 
neighbourhood of $\partial(S D)$ in $S D$, and $\left.u\right|_{\partial(S D)}=0$. Then the integral identity (28) is valid for $u$.

Proof. We want to construct a sequence of smooth functions $u_{k}$ coinciding with $u$ in a neighbourhood of $\partial(S D)$ and such that $u_{k} \rightarrow u$ in $H^{1}(S D)$ and $\mathbf{F} u_{k} \rightarrow \mathbf{F} u$ in $H^{1}(S D)$ as $k \rightarrow \infty$. Then applying (2.3) to each function $u_{k}$ and passing to the limit as $k \rightarrow \infty$, we come to the desired conclusion.

A chart $(O, \varphi)$ of the manifold $S N$ is called straightening the vector field $\mathbf{F}$ if $\psi_{*} \mathbf{F}$ coincides with a coordinate vector field on the range $\psi(O) \subset \mathbb{R}^{2}$. Cover $S D$ by an atlas consisting of sufficiently small straightening charts $\left(O_{i}, \varphi_{i}\right)$, so that the charts having nonempty intersection with $\partial(S D)$ lie entirely in that neighbourhood of $\partial(S D)$ where $u$ is smooth. Choose a partition of unity $\left\{\mu_{i}\right\}$ subordinate to this atlas. Then $u=\sum_{i} \mu_{i} u$, where each $\mu_{i} u$ has support in $O_{i}$.

Choose those indices $i$ for which $O_{i}$ is disjoint from $\partial(S D)$. Fix a nonnegative function $\kappa \in C_{0}^{\infty}\left(\mathbb{R}^{2}\right)$ such that $\int_{\mathbb{R}^{2}} \kappa d x=1$. Set $\kappa_{\delta}(x)=\kappa(x / \delta) / \delta^{2}$ for $\delta>0$. The function $\tilde{u}_{i}^{\delta}=\left(\left(\mu_{i} u\right) \circ \varphi_{i}^{-1}\right) * \kappa_{\delta}$ is $C^{\infty}$-smooth on $\mathbb{R}^{2}$ and $\operatorname{supp} \tilde{u}_{i}^{\delta} \subset \varphi_{i}\left(O_{i}\right)$ for $\delta>0$ small enough. The difference $\tilde{u}_{i}^{\delta}-\left(\mu_{i} u\right) \circ \varphi_{i}^{-1}$ tends to zero uniformly on $\mathbb{R}^{2}$ as $\delta \rightarrow 0$. Lift the function $\tilde{u}_{i}^{\delta}$ to $O_{i}$ by $\varphi_{i}$, i.e. consider the function $\tilde{u}_{i}^{\delta} \circ \varphi_{i}$ and denote it again by $\tilde{u}_{i}^{\delta}$. For the other indices, take the functions $\mu_{i} u$ themselves for $\tilde{u}_{i}^{\delta}$. Then the sum $u_{\delta}=\sum_{i} \tilde{u}_{i}^{\delta}$ is the sought approximation to $u$.

3.4. Riccati equation and second integral identity. For $(x, v) \in S M$, define

$$
\mathcal{V}(x, v):=\operatorname{ker} d_{(x, v)} \pi, \quad \text { and } \quad E(x, v):=\mathcal{V}(x, v) \oplus \mathbb{R} \mathbf{F}(x, v),
$$

where $d_{(x, v)} \pi$ is the differential of the natural projection $\pi: S M \rightarrow M$ and $\mathbf{F}$ is the infinitesimal generator of the thermostat flow.

Lemma 3.6. If $\gamma:[0, T] \rightarrow M$ is a $\lambda$-geodesic, then

$$
d_{\dot{\gamma}(0)} \phi_{t}(E) \cap \mathcal{V}(\gamma(t), \dot{\gamma}(t))=\{0\}
$$

for every $t \in(0, T]$.

Proof. Take $(x, v) \in S M$ and $t \in(0, T]$. From the definition of $\exp ^{\lambda}$ it is straightforward that

$$
\operatorname{image}\left(d_{t v} \exp _{x}^{\lambda}\right)=d_{\dot{\gamma}(t)} \pi\left(d_{\dot{\gamma}(0)} \phi_{t}(E)\right) .
$$

By the absence of conjugate points, $d_{w} \exp _{x}^{\lambda}$ is a linear isomorphism for every $w \in T_{x} M$ at which $\exp _{x}^{\lambda}$ is defined, and the lemma follows.

For $(x, v) \in S M$ there is $t_{0}$ such that $x_{0}=\gamma_{x, v}\left(t_{0}\right) \in U \backslash M$. If $x_{0}$ is close enough to $M$, then $\gamma_{x_{0}, v_{0}}$ has no conjugate points either, where 
$v_{0}=\dot{\gamma}_{x, v}\left(t_{0}\right)$. Then Lemma 3.6 implies that there is a unique continuous function $r(t)=r\left(\phi_{t}\left(x_{0}, v_{0}\right)\right)$ along the orbit of $\left(x_{0}, v_{0}\right)$ such that

$$
H\left(\phi_{t}\left(x_{0}, v_{0}\right)\right)+r(t) V\left(\phi_{t}\left(x_{0}, v_{0}\right)\right) \in d_{v_{0}} \phi_{t}(E) .
$$

In this way we can define $r$ on the whole $S M$. The so-obtained function $r$ is smooth on every orbit and $r \in L^{\infty}(S M)$. Below we will need to use the fact that the function $r$ satisfies a Riccati type equation along the flow.

Lemma 3.7. (Riccati equation). The function $r$ satisfies

$$
\mathbf{F}(r-V(\lambda))+r(\lambda I-V(\lambda)+r)+\mathbb{K}-\lambda I V(\lambda)=0 .
$$

Proof. Fix $(x, v) \in S M$ and set

$$
\xi(t):=d \phi_{-t}\left(H\left(\phi_{t}(x, v)\right)+r\left(\phi_{t}(x, v)\right) V\left(\phi_{t}(x, v)\right)\right) .
$$

By the definition of $r, \xi(t) \in E(x, v)$ for all $t$. Differentiating with respect to $t$ and setting $t=0$ we obtain:

$$
\dot{\xi}(0)=[\mathbf{F}, H]+\mathbf{F}(r) V+r[\mathbf{F}, V] .
$$

Using the commutation relations (7) we have

$$
\dot{\xi}(0)=-\lambda \mathbf{F}-\lambda I \xi(0)+\left\{K-H(\lambda)-\lambda J+\lambda^{2}+\mathbf{F}(r)-r V(\lambda)\right\} V .
$$

Replacing $H$ by $\xi(0)-r V$ yields:

$\dot{\xi}(0)+(r+\lambda I) \xi(0)-\lambda \mathbf{F}=\left\{K-H(\lambda)-\lambda J+\lambda^{2}+\mathbf{F}(r)+\lambda I r-r V(\lambda)+r^{2}\right\} V$.

Since $\dot{\xi}(0)+(r+\lambda I) \xi(0)-\lambda \mathbf{F} \in E$ we must have

$$
K-H(\lambda)-\lambda J+\lambda^{2}+\mathbf{F}(r)+\lambda I r-r V(\lambda)+r^{2}=0
$$

which is the desired equation.

For the proof of Theorem 1.1 we also need the following result.

Theorem 3.8. Let $\varphi \rightarrow \mathbb{R}$ be a function vanishing on $\partial(S M)$ such that $\varphi \in C^{\infty}(R M)$. Then

$$
\int_{S M}(\mathbf{F} \varphi)^{2} d \mu-\int_{S M} \mathbb{K} \varphi^{2} d \mu=\int_{S M}[\mathbf{F}(\varphi)-r \varphi+\varphi V(\lambda)]^{2} d \mu \geq 0 .
$$

Moreover,

$$
\int_{S M}[\mathbf{F}(\varphi)-r \varphi+\varphi V(\lambda)]^{2} d \mu=0
$$

if and only if $\varphi=0$ on $R M$. 
Proof. Let us expand $[\mathbf{F}(\varphi)-r \varphi+\varphi V(\lambda)]^{2}$ :

$$
\begin{aligned}
{[\mathbf{F}(\varphi)-r \varphi+\varphi V(\lambda)]^{2} } & =[\mathbf{F}(\varphi)]^{2}+\varphi^{2} r^{2}+\varphi^{2}[V(\lambda)]^{2} \\
& -2 \mathbf{F}(\varphi) \varphi r+2 \mathbf{F}(\varphi) \varphi V(\lambda)-2 \varphi^{2} r V(\lambda) .
\end{aligned}
$$

Using Lemma 3.7, we obtain:

$$
\begin{aligned}
{[\mathbf{F}(\varphi)-r \varphi+\varphi V(\lambda)]^{2} } & =[\mathbf{F}(\varphi)]^{2}-\mathbb{K} \varphi^{2} \\
& -\mathbf{F}\left((r-V(\lambda)) \varphi^{2}\right)+\varphi^{2}[V(\lambda)]^{2} \\
& -\varphi^{2} r[\lambda I+V(\lambda)]+\lambda I V(\lambda) \varphi^{2}
\end{aligned}
$$

If we integrate the last equality with respect to the measure $\mu$, we obtain as desired:

$$
\int_{S M}(\mathbf{F} \varphi)^{2} d \mu-\int_{S M} \mathbb{K} \varphi^{2} d \mu=\int_{S M}[\mathbf{F}(\varphi)-r \varphi+\varphi V(\lambda)]^{2} d \mu
$$

since by the Stokes Theorem and (8), we have

$$
\begin{array}{r}
\int_{S M} \mathbf{F}\left((r-V(\lambda)) \varphi^{2}\right) d \mu=\int_{S M}\left\{\varphi^{2}[V(\lambda)]^{2}-\varphi^{2} r[\lambda I+V(\lambda)]+\lambda I V(\lambda) \varphi^{2}\right\} d \mu \\
+\int_{\partial(S M)}(r-V(\lambda)) \varphi^{2} i_{\mathbf{F}} \Theta
\end{array}
$$

while the last integral vanishes due to the boundary condition.

Suppose now

$$
\int_{S M}[\mathbf{F}(\varphi)-r \varphi+\varphi V(\lambda)]^{2} d \mu=0
$$

which implies

$$
\mathbf{F}(\varphi)-r \varphi+\varphi V(\lambda)=0
$$

on $R M$. This means that, on almost every orbit, the function $\varphi$ satisfies a homogeneous first-order ordinary differential equation with zero boundary data. This surely implies that $\varphi \equiv 0$ on such an orbit, which yelds $\varphi \equiv 0$ on $R M$.

3.5. End of the proof of Theorem 1.1. Let $W \subset N$ be a collar neighbourhood of $\partial M$ in $N$. This means that there is a diffeomorphism $\Psi$ : $\partial M \times(-1,1) \rightarrow W$ such that the restriction $\left.\Psi\right|_{\partial M \times\{0\}}$ is the identity map. We also assume that $\Psi(\partial M \times(-1,0)) \subset M$ and $\Psi(\partial M \times(0,1)) \subset N \backslash M$.

Put $M_{\varepsilon}=M \cup \Psi(\partial M \times[0, \varepsilon]), 0 \leq \varepsilon<1$, obtaining a nested family of subdomains in $N$, with $M_{0}=M$.

Let us now prove Theorem 1.1. By Lemma 3.4 the function $u$ satisfies the condition of Lemma 3.5 with $D=M_{\varepsilon}$ for every $\varepsilon>0$. Then by Lemma 3.5 
we have

$$
\int_{S M_{\varepsilon}}(\mathbf{F} V u)^{2} d \mu-\int_{S M_{\varepsilon}} \mathbb{K}(V u)^{2} d \mu=\int_{S M_{\varepsilon}}(V \mathbf{F} u)^{2} d \mu-\int_{S M_{\varepsilon}}(\mathbf{F} u)^{2} d \mu
$$

for every $\varepsilon>0$. It is easy to see that the right-hand side of the last equation is nonpositive. Indeed, since $V \mathbf{F} u=-V \alpha(x, v)$ we have

$$
\int_{S M_{\varepsilon}} f \alpha d \mu=0 \text { and } \int_{S M_{\varepsilon}} \alpha^{2} d \mu=\int_{S M_{\varepsilon}}(V \alpha)^{2} d \mu \text {. }
$$

This follows from [7, Lemma 4.4], which holds in any dimension. Thus

$$
\int_{S M_{\varepsilon}}(\mathbf{F} V u)^{2} d \mu-\int_{S M_{\varepsilon}} \mathbb{K}(V u)^{2} d \mu=-\int_{S M_{\varepsilon}} f^{2} d \mu \leq 0 .
$$

Lemma 3.4 allows us to pass to the limit as $\varepsilon \rightarrow 0$ in this identity. Using Theorem 3.8 we obtain that $V u \equiv 0$ on $R M$. This says that $u=-h$ almost everywhere for some $h \in C_{0}^{\infty}(M)$. Since $u \in C(S M)$, then $u=-h$ everywhere. But in this case, since $d \pi_{(x, v)}(\mathbf{F})=v$ we have $\mathbf{F} u=-d h_{x}(v)$. This clearly implies the claim of Theorem 1.1.

\section{Proof of Theorem 1.5}

The considerations here generalize mostly those in [6], where the Riemannian case was treated. We start Subsection 4.1 with derivation of the Riccati equation. In Subsection 4.2 we obtain from it a certain integral identity. The latter, combined with the Pestov integral identity, will prove Theorem 1.5 in Subsection 4.3. Subsection 4.4 plays an auxiliary role and is devoted to providing a sufficient condition for a thermostat flow to be Anosov on using the hyperbolicity test due to M. Wojtkowski [24].

4.1. Riccati equation for Anosov thermostats. Recall that the Anosov property means that there is a continuous invariant splitting $T(S M)=\mathbb{R} \mathbf{F} \oplus$ $E^{u} \oplus E^{s}$ in such a way that there are constants $C>0$ and $0<\rho<1<\eta$ such that for all $t>0$ we have

$$
\left\|\left.d \phi_{-t}\right|_{E^{u}}\right\| \leq C \eta^{-t} \text { and }\left\|\left.d \phi_{t}\right|_{E^{s}}\right\| \leq C \rho^{t},
$$

where the norms are taken with respect to a Sasaki type Riemannian metric on $S M$ induced by the Finsler metric $F$. The subbundles are then invariant and Hölder continuous and have smooth integral manifolds, the stable and unstable manifolds, which define a continuous foliation with smooth leaves.

Let us introduce the weak stable and unstable bundles:

$$
\begin{aligned}
& E^{+}=\mathbb{R} \mathbf{F} \oplus E^{s}, \\
& E^{-}=\mathbb{R} \mathbf{F} \oplus E^{u} .
\end{aligned}
$$

Lemma 4.1. For any $(x, v) \in S M, V(x, v) \notin E^{ \pm}(x, v)$. 
Proof. Let $\Lambda(S M)$ be the bundle above $S M$ that at each point $(x, v) \in S M$ consists of all 2-dimensional subspaces $W$ of $T_{(x, v)} S M$ with $\mathbf{F}(x, v) \in W$.

The map $(x, v) \mapsto \mathbb{R} \mathbf{F}(x, v) \oplus \mathbb{R} V(x, v)$ is a section of $\Lambda(S M)$ and its image is a codimension one submanifold that we denote by $\Lambda_{V}$ and call it Maslov cycle. Similarly the map $(x, v) \mapsto \mathbb{R} \mathbf{F}(x, v) \oplus \mathbb{R} H(x, v)$ is a section of $\Lambda(S M)$ and its image is a codimension one submanifold that we denote by $\Lambda_{H}$.

The flow $\phi$ naturally lifts to a flow $\phi^{*}$ acting on $\Lambda(S M)$ via its differential. Let $\mathbf{F}^{*}$ be the infinitesimal generator of $\phi^{*}$.

Claim 4.2. $\mathbf{F}^{*}$ is transversal to the Maslov cycle $\Lambda_{V}$.

Proof. Indeed, define a function $m: \Lambda(S M) \backslash \Lambda_{H} \rightarrow \mathbb{R}$ as follows. If $W \in$ $\Lambda(S M) \backslash \Lambda_{H}$, then $H \notin W$. Thus there exists a unique $m=m(W)$ such that $m H+V \in W$. Clearly $m$ is smooth and $\Lambda_{V}=m^{-1}(0) \subset \Lambda(S M) \backslash \Lambda_{H}$. Fix $(x, v) \in S M$ and set

$$
m(t):=m\left(\phi_{t}^{*}(\mathbb{R} F(x, v) \oplus \mathbb{R} V(x, v))\right) .
$$

By the definition of $m$, there exist functions $x(t)$ and $y(t)$ such that

$$
m(t) H\left(\phi_{t}(x, v)\right)+V\left(\phi_{t}(x, v)\right)=x(t) \mathbf{F}\left(\phi_{t}(x, v)\right)+y(t) d \phi_{t}(V(x, v)) .
$$

Equivalently

$$
m(t) d \phi_{-t}\left(H\left(\phi_{t}(x, v)\right)\right)+d \phi_{-t}\left(V\left(\phi_{t}(x, v)\right)\right)=x(t) \mathbf{F}(x, v)+y(t) V(x, v) .
$$

Differentiating with respect to $t$ and setting $t=0$ (recall that $m(0)=0$ ) we obtain:

$$
\dot{m}(0) H+[\mathbf{F}, V]=\dot{x}(0) \mathbf{F}+\dot{y}(0) V .
$$

But $[V, \mathbf{F}]=H+V(\lambda) V$. Thus $\dot{m}(0)=1$ which proves the claim.

From the Claim 4.2 it follows that $\Lambda_{V}$ determines an oriented codimension one cycle in $\Lambda(S M)$ and by duality it defines a cohomology class $\mathfrak{m} \in$ $H^{1}(\Lambda(S M), \mathbb{Z})$. Set $E=E^{ \pm}$. Given a continuous closed curve $\alpha: S^{1} \rightarrow S M$, the index of $\alpha$ is $\nu(\alpha):=\langle\mathfrak{m},[E \circ \alpha]\rangle$ (i.e. $\nu=E^{*} \mathfrak{m} \in H^{1}(S M, \mathbb{Z})$ ). The index of $\alpha$ only depends on the homology class of $\alpha$. Since $E$ is $\phi$-invariant, the Claim 4.2 also ensures that if $\gamma$ is any closed orbit of $\phi$, then $\nu(\gamma) \geq 0$.

Recall that according to Ghys [12] we know that $\phi$ is topologically conjugate to the geodesic flow of a metric of constant negative curvature. In particular, every homology class in $H_{1}(S M, \mathbb{Z})$ contains a closed orbit of $\phi$. Thus $\nu$ must vanish.

If there exists $(x, v) \in S M$ for which $V(x, v) \in E(x, v)$, then using that every point of $\phi$ is non-wandering, we can produce exactly as in [19. Lemma 2.49] a closed curve $\alpha: S^{1} \rightarrow S M$ with $\nu(\alpha)>0$. This contradiction shows the lemma. 
Lemma 4.1 implies that there exist unique continuous functions $r^{ \pm}(x, v)$ on $S M$ such that

$$
\begin{aligned}
& H(x, v)+r^{+}(x, v) V(x, v) \in E^{+}, \\
& H(x, v)+r^{-}(x, v) V(x, v) \in E^{-} .
\end{aligned}
$$

Note that the Anosov property implies that $r^{ \pm}$are smooth along $\phi$ and $r^{+} \neq r^{-}$everywhere. Next, we show that the functions $r^{ \pm}$satisfy a Riccati type equation along the flow.

Lemma 4.3. The function $r=r^{ \pm}$satisfies

$$
\mathbf{F}(r-V(\lambda))+r(\lambda I-V(\lambda)+r)+\mathbb{K}-\lambda I V(\lambda)=0 .
$$

Proof. Let $E=E^{ \pm}$. Fix $(x, v) \in S M$ and set

$$
\xi(t):=d \phi_{-t}\left(H\left(\phi_{t}(x, v)\right)+r\left(\phi_{t}(x, v)\right) V\left(\phi_{t}(x, v)\right)\right) .
$$

By the definition of $r, \xi(t) \in E(x, v)$ for all $t$. Differentiating with respect to $t$ and setting $t=0$ we obtain:

$$
\dot{\xi}(0)=[\mathbf{F}, H]+\mathbf{F}(r) V+r[\mathbf{F}, V] .
$$

Using the commutation relations (7) we have

$$
\dot{\xi}(0)=-\lambda \mathbf{F}-\lambda I \xi(0)+\left\{K-H(\lambda)-\lambda J+\lambda^{2}+\mathbf{F}(r)-r V(\lambda)\right\} V .
$$

Replacing $H$ by $\xi(0)-r V$ yields:

$\dot{\xi}(0)+(r+\lambda I) \xi(0)-\lambda \mathbf{F}=\left\{K-H(\lambda)-\lambda J+\lambda^{2}+\mathbf{F}(r)+\lambda I r-r V(\lambda)+r^{2}\right\} V$.

Since $\dot{\xi}(0)+(r+\lambda I) \xi(0)-\lambda \mathbf{F} \in E$ we must have

$$
K-H(\lambda)-\lambda J+\lambda^{2}+\mathbf{F}(r)+\lambda I r-r V(\lambda)+r^{2}=0
$$

which is the desired equation.

4.2. Second integral identity for Anosov thermostats. Here we prove the following integral identity which we use in the proof of Theorem 1.3.

Theorem 4.4. Let $\varphi: S M \rightarrow \mathbb{R}$ be a smooth function and suppose the flow $\phi_{t}$ is Anosov. Then for $r=r^{ \pm}$

$$
\int_{S M}(\mathbf{F} \varphi)^{2} d \mu-\int_{S M} \mathbb{K} \varphi^{2} d \mu=\int_{S M}[\mathbf{F}(\varphi)-r \varphi+\varphi V(\lambda)]^{2} d \mu \geq 0 .
$$

Moreover,

$$
\int_{S M}[\mathbf{F}(\varphi)-r \varphi+\varphi V(\lambda)]^{2} d \mu=0
$$

if and only if $\varphi=0$. 
Proof. We omit the proof of the first part since it is exactly the same as the first part of Theorem 3.8. Suppose now

$$
\int_{S M}[\mathbf{F}(\varphi)-r \varphi+\varphi V(\lambda)]^{2} d \mu=0
$$

which implies

$$
\mathbf{F}(\varphi)-r \varphi+\varphi V(\lambda)=0
$$

everywhere. Since this holds for $r=r^{ \pm}$, we deduce:

$$
\left(r^{+}-r^{-}\right) \psi=0 \text {. }
$$

But for an Anosov flow $r^{+}-r^{-} \neq 0$. This surely implies that $\varphi \equiv 0$ on $S M$.

4.3. End of the proof of Theorem 1.5. Assume we have a cohomological equation

$$
\mathbf{F} u(x, v)=f(x)+\alpha_{x}(v) .
$$

By the Pestov integral identity we have

$$
\int_{S M}(\mathbf{F} V u)^{2} d \mu-\int_{S M} \mathbb{K}(V u)^{2} d \mu=\int_{S M}(V \mathbf{F} u)^{2} d \mu-\int_{S M}(\mathbf{F} u)^{2} d \mu .
$$

It is easy to see that the right-hand side of the last equation is nonpositive. Indeed, since $V \mathbf{F} u=V \alpha(x, v)$ we have

$$
\int_{S M} f \alpha d \mu=0 \text { and } \int_{S M} \alpha^{2} d \mu=\int_{S M}(V \alpha)^{2} d \mu .
$$

This follows from [7, Lemma 4.4], which holds in any dimension. Thus

$$
\int_{S M}(\mathbf{F} V u)^{2} d \mu-\int_{S M} \mathbb{K}(V u)^{2} d \mu=-\int_{S M} f^{2} d \mu \leq 0 .
$$

Using Theorem 4.4 we obtain that $V u \equiv 0$ on $S M$, which says that $u=h$ some $h \in C^{\infty}(M)$. Since $d \pi_{(x, v)}(\mathbf{F})=v$ we have $\mathbf{F} u=d h_{x}(v)$. This clearly implies the claim of Theorem 1.5 .

\subsection{Sufficient condition for a thermostat flow to be Anosov.}

Theorem 4.5. If $(M, F, \lambda)$ is a thermostat on a closed oriented Finsler surface and

$$
K-H(\lambda)-\lambda J+\lambda^{2}+\frac{(\lambda I+V(\lambda))^{2}}{4}<0,
$$

then the flow $\phi_{t}$ is Anosov. 
Proof. We apply the hyperbolicity test of [24, Theorem 5.2].

Given $\xi \in T_{(x, v)}(S M)$ we may write:

$$
\xi=a \mathbf{F}(x, v)+y H(x, v)+z V(x, v) .
$$

Define a quadratic form $\mathbb{Q}$ on $S M$ by

$$
\mathbb{Q}(\xi)=y z .
$$

Consider the quotient bundle $\hat{T}(S M)$ defined by

$$
\hat{T}_{(x, v)}(S M):=T_{(x, v)}(S M) / \mathbb{R} \mathbf{F}(x, v) .
$$

Since $d \phi_{t} \mathbf{F}(x, v)=\mathbf{F}\left(\phi_{t}(x, v)\right)$, the differential $d \phi_{t}$ descends to the quotient to define a map $A_{t}: \hat{T}_{(x, v)}(S M) \rightarrow \hat{T}_{\phi_{t}(x, v)}(S M)$ satisfying

$$
A_{s+t}=A_{s} \circ A_{t} \text {. }
$$

By Theorem 5.2 of [24], it suffices to prove that the flow $\phi_{t}$ is strictly monotone with respect to the quadratic form $\mathbb{Q}$, i.e., the projection of the Lie derivative $\mathcal{L}_{\mathbf{F}} \mathbb{Q}$ onto the quotient bundle is positive definite. To this end, we need the following lemma.

Lemma 4.6. For $\xi \in T_{(x, v)} S M$ consider the representation of $d \phi_{t}(\xi)$ in terms of $\mathbf{F}, H, V$ :

$$
d \phi_{t}(\xi)=a(t) \mathbf{F}\left(\phi_{t}(x, v)\right)+y(t) H\left(\phi_{t}(x, v)\right)+z(t) V\left(\phi_{t}(x, v)\right) .
$$

Then the functions $a, y, z$ satisfy the following equations

$$
\begin{aligned}
& \dot{a}=\lambda y, \\
& \dot{y}=\lambda I y+z, \\
& \dot{z}=-\left\{K-H(\lambda)-\lambda J+\lambda^{2}\right\} y+V(\lambda) z .
\end{aligned}
$$

Proof. Equivalently we have

$$
\xi=a(t) d \phi_{-t}\left(\mathbf{F}\left(\phi_{t}(x, v)\right)\right)+y(t) d \phi_{-t}\left(H\left(\phi_{t}(x, v)\right)\right)+z(t) d \phi_{-t}\left(V\left(\phi_{t}(x, v)\right)\right) .
$$

If we differentiate the last equality with respect to $t$ we obtain:

$$
0=\dot{a} \mathbf{F}+\dot{y} H+y[\mathbf{F}, H]+\dot{z} V+z[\mathbf{F}, V] .
$$

Using the commutation relations (7) and regrouping, we find out the equations of Lemma 4.6.

Continuing the prof of the theorem, we have

$$
\begin{aligned}
\left.\mathcal{L}_{\mathbf{F}} \mathbb{Q}\right|_{\hat{T}(S M)} & =\left.\frac{d}{d t}\right|_{t=0} \hat{\mathbb{Q}}\left(A_{t}(\xi)\right)=\left.\frac{d}{d t}\right|_{t=0}(y(t) z(t)) \\
& =-\left\{K-H(\lambda)-\lambda J+\lambda^{2}\right\} y^{2}+(V(\lambda)+\lambda I) y z+z^{2} .
\end{aligned}
$$


By Sylvester's criterion it is positively definite if and only if

$$
K-H(\lambda)-\lambda J+\lambda^{2}+\frac{(\lambda I+V(\lambda))^{2}}{4}<0,
$$

which concludes the proof of the theorem.

\section{Proof of Theorem 1.6}

In Subsection 5.1 we give an equivalent condition to the absence of conjugate points for a thermostat in terms of the Jacobi equation. This is completely analogous to the case of the geodesic flow. Subsection 5.2 is devoted to constructing an integrable solution of the corresponding Riccati equation. This is achieved by reducing the problem to Hopf's construction in [14] and is similar to the considerations we used before in [1, Sections 4-5]. Finally, in Subsection 5.3 we conclude the proof of Theorem 1.6 by applying the Pestov integral identity.

\subsection{Thermostats without conjugate points. Put}

$$
q=-\lambda I-V(\lambda), \quad k=\mathbb{K}-\mathbf{F} V(\lambda)-\mathbf{F}(\lambda I) .
$$

Our aim in this subsection is the following

Theorem 5.1. A thermostat $(M, F, \lambda)$ has no conjugate points if and only if all solutions of the Jacobi equation

$$
\ddot{y}+q \dot{y}+k y=0
$$

along any $\lambda$-geodesic, vanish at most once.

We consider a variation of the $\lambda$-geodesic $\gamma(t)=\pi \circ \phi_{t}(x, v)$ for some $(x, v) \in S M$. We take the variation $c(s, t)=\pi\left(\phi_{t}(Z(s))\right)$ of $\gamma$ that depends on a curve $Z \subset T M$ with $\dot{Z}(0)=\xi \in T_{(x, v)} S M$. The vector field $J_{\xi}(t):=$ $\left.\frac{\partial c}{\partial s}\right|_{s=0}(t)$ (that depends on $\xi$ ) is called a Jacobi field along $\gamma$.

Lemma 5.2. Every Jacobi field $J_{\xi}$, expressed as

$$
J_{\xi}(t)=x(t) \dot{\gamma}(t)+y(t) i \dot{\gamma}(t),
$$

satisfies the Jacobi equations

$$
\begin{aligned}
& \dot{x}=\lambda y, \\
& \ddot{y}+q \dot{y}+k y=0 .
\end{aligned}
$$

In particular, if a Jacobi field $J$ is tangent to the $\lambda$-geodesic $\gamma$ everywhere, then $J=c \gamma$, where $c=$ const. 
Proof. For $\xi \in T(S M)$, write

$$
d \phi_{t}(\xi)=x(t) \mathbf{F}+y(t) H+z(t) V
$$

equivalently

$$
\xi=x(t) d \phi_{-t}(\mathbf{F})+y(t) d \phi_{-t}(H)+z(t) d \phi_{-t}(V) .
$$

If we differentiate the last equality with respect to $t$, we obtain:

$$
0=\dot{x} \mathbf{F}+\dot{y} H+y[\mathbf{F}, H]+\dot{z} V+z[\mathbf{F}, V] .
$$

Using the bracket relations and regrouping we arrive at (31) and (32).

Let $\gamma:[0, T] \rightarrow M$ be a $\lambda$-geodesic with endpoints $x=\gamma(0)$ and $y=\gamma(T)$. We say that $x$ and $y$ are conjugate along $\gamma$ if the map $d_{T \dot{\gamma}(0)} \exp _{x}^{\lambda}$ has a nonmaximal rank. Note that this definition does not contradict the definition of the absence of conjugate points mentioned in the Introduction.

There exists a simple but very useful and well-known relation between the exponential map and the Jacobi fields:

Theorem 5.3. Let $\gamma:[0, T] \rightarrow M$ be a $\lambda$-geodesic with endpoints $x=\gamma(0)$ and $y=\gamma(T)$. Then $x$ and $y$ are conjugate along $\gamma$ if and only if there exists a nonzero Jacobi field along $\gamma$ satisfying $J(0)=J(T)=0$.

Proof. For the proof we need the following

Lemma 5.4. Let $\gamma:[0, T] \rightarrow M$ be a $\lambda$-geodesic such that $\gamma(t)=\exp _{x}^{\lambda}(t v)$, with $x=\gamma(0)$ and $v=\dot{\gamma}(0)$. Let $w \in T_{x} M$. Then $J(t)=d_{t v} \exp _{x}^{\lambda}(t w)$ is a Jacobi field along $\gamma$. Moreover, $J(0)=0$ and $D_{t} J(0)=w$.

The proof of the lemma is standard and we give it for completeness after the proof of theorem.

Suppose there exists a nonzero vector $w \in T_{x} M$ such that $d_{v} \exp _{x}^{\lambda}(w)=0$. By Lemma 5.4, the Jacobi field $J(t)=d_{t v} \exp _{x}^{\lambda}\left(t T^{-1} w\right)$ is nontrivial, since $D_{t} J(0)=T^{-1} w \neq 0$, and it satisfies $J(0)=J(T)=0$.

Conversely, if the points $x$ and $y$ are conjugate along $\gamma$ then there exists a nontrivial Jacobi field $J$ along $\gamma$ such that $J(0)=J(T)=0$. Let $D_{t} J(0)=$ $w$. Then $w \neq 0$ and by Lemma $5.4 d_{T \dot{\gamma}(0)} \exp _{x}^{\lambda}(T w)=J(T)=0$ so that $y=\exp _{x}^{\lambda}(T \dot{\gamma}(0))$. This means that $w \in \operatorname{ker} d_{T \dot{\gamma}(0)} \exp _{x}^{\lambda}$.

Proof of Lemma 5.4. Consider the variation $c(s, t)=\exp _{x}^{\lambda}(t(v+s w))$ of $\gamma$. Since

$$
\frac{\partial c}{\partial s}(s, t)=\frac{\partial \exp _{x}^{\lambda}(t(v+s w))}{\partial s}=d_{t(v+s w)} \exp _{x}^{\lambda}(t w),
$$

the vector field $J(t)$ is a Jacobi field. Once $d_{0} \exp _{x}^{\lambda}$ is the identity map, we have

$$
J(0)=d_{0} \exp _{x}^{\lambda}(0)=0 .
$$


It is well known that $D_{s} Y(s, t)=D_{t} J(s, t)$, where $Y(s, t)=\frac{\partial c}{\partial t}(s, t)$ and $J(s, t)=\frac{\partial c}{\partial s}(s, t)$. Then

$$
D_{t} J(s, 0)=D_{s} Y(s, 0)=D_{s}\left(d_{0} \exp _{x}^{\lambda}(v+s w)\right)=\frac{\partial(v+s w)}{\partial s}=w,
$$

which finishes the proof.

Let $\xi \in T_{(x, v)} T M$, and let $Z(t)=(\alpha(t), z(t))$ be any curve with $Z(0)=$ $(x, v)$ and $\dot{Z}(0)=\xi$. Define the connection map

$$
\mathcal{K}_{(x, v)}(\xi):=\nabla_{\dot{\alpha}} \dot{z}(0) .
$$

For $(x, v) \in T M$, define the horizontal subbundle by $\mathcal{H}(x, v)=\operatorname{ker} \mathcal{K}_{(x, v)}$. So we obtain the following isomorphism:

$$
T_{(x, v)} T M \rightarrow \mathcal{H}(x, v) \oplus \mathcal{V}(x, v), \quad \xi \mapsto\left(d \pi_{(x, v)}(\xi), \mathcal{K}_{(x, v)}(\xi)\right) .
$$

Proof of Theorem 5.1. Assume that a thermostat has no conjugate points. Let $\gamma(t), 0 \leq t \leq T$, be a $\lambda$-geodesic. By Lemma [3.6, $d_{\dot{\gamma}(0)} \phi_{t}(E)$ can be seen to be a graph over the horizontal subspace for $t \in(0, T]$. We may thus express

$$
d_{\dot{\gamma}(0)} \phi_{t}(E)=\operatorname{graph} S:=\{(v, S(t) v), v \in \mathcal{H}(\dot{\gamma}(t)\}
$$

with some $S(t): \mathcal{H}(\dot{\gamma}(t)) \rightarrow \mathcal{V}(\dot{\gamma}(t))$ for $t \in(0, T]$. We have (see, for instance, [15. Lemma 3.1])

$$
d \phi_{t}(\xi)=\left(J_{\xi}(t), \dot{J}_{\xi}(t)\right)
$$

Let $u(t):=\langle S(t) i \dot{\gamma}, i \dot{\gamma}\rangle$. In view of (33) $), \dot{J}_{\eta}=S J_{\eta}$ for all $\eta \in T_{(x, v)} S M$. Using all of this, we obtain that $\dot{y}=u y$. Since $u$ is well defined for all $t \in(0, T]$, it is easy to see that $y$ never vanishes for $t \in(0, T]$.

Conversely, suppose that, for any $\lambda$-geodesic $\gamma(t)$, any solution of (30) on $\gamma$ with $y(a)=y(b)=0$ for some $a<b$ is necessarily $y \equiv 0$. Let $J(t)$ be a Jacobi field along $\gamma$ with $J(a)=J(b)=0$. Using Lemma 5.2 we then conclude that $J(t)$ is of the form $J=c \dot{\gamma}$. As soon as $J(a)=J(b)=0, J$ vanishes identically. Appealing to Theorem 5.3, we conclude the proof.

5.2. Riccati equation. Let $\gamma(t),-\infty<t<+\infty$, be a complete unit speed $\lambda$-geodesic. The Jacobi equation on $\gamma$ is:

$$
\ddot{y}+q \dot{y}+k y=0 .
$$

If $y(t)$ is a nowhere vanishing solution of (34), then $r(t)=\frac{\dot{y}(t)}{y(t)}$ is a solution of the Riccati equation

$$
\dot{r}+r^{2}+q r+k=0 .
$$

Let

$$
m(t):=\exp \left(-\frac{1}{2} \int q(t) d t\right)
$$


If $y(t)=m(t) z(t)$ then $z(t)$ is a solution of the equation

$$
\ddot{z}+\tilde{k} z=0 \text {, }
$$

where

$$
\tilde{k}(t)=k(t)-\frac{\dot{q}}{2}+\frac{q^{2}}{4} .
$$

Since $m(t)$ is nowhere zero, equation (34) has no conjugate points if and only if so does equation (36).

The Riccati equation corresponding to ( $\underline{36}$ ) is

$$
\dot{u}+u^{2}+\tilde{k}=0 .
$$

Clearly, the solutions of (35) and (38) are related by

$$
r(t)=u(t)-q(t) / 2 .
$$

Observe that, once $S M$ is compact, there is a constant $A \geq 0$ such that

$$
|\tilde{k}(x, v)|=\left|k(x, v)-\frac{F(q(x, v))}{2}+\frac{q^{2}(x, v)}{4}\right| \leq A^{2}
$$

for all $(x, v) \in S M$. Since $\tilde{k}(t)$ is the restriction of $\tilde{k}(x, v)$ to $(\gamma, \dot{\gamma})$, we have

$$
|\tilde{k}(t)| \leq A^{2} \text {. }
$$

In 14, Hopf constructed a solution $u(t)$ of (38) such that $|u(t)| \leq A$ for all $t$. Considering all $\gamma$ gives a bounded function $u(x, v)$ on $S M$ whose resctriction to any $\gamma$ is a solution of (38), and Hopf proves in [14] that this $u(x, v)$ is a measurable function on $S M$. In view of (39), taking $r(x, v)=$ $u(x, v)-q(x, v) / 2$ then yields a bounded measurable function $r(x, v)$ whose restriction to any $\gamma$ is a solution of (35). From (35) we readily infer that $r(x, v)$ satisfies the following equation on $S M$ :

$$
\mathbf{F}(r)+r^{2}+q r+k=0 .
$$

5.3. End of the proof of Theorem 1.6. Using the same arguments as in the proof of Theorem 2.3, we can derive the following integral identity for any $\varphi \in C^{\infty}(S M)$ :

$$
\int_{S M}(\mathbf{F} \varphi)^{2} d \mu-\int_{S M} \mathbb{K} \varphi^{2} d \mu=\int_{S M}[\mathbf{F}(\varphi)-r \varphi+\varphi(\lambda I+V(\lambda))]^{2} d \mu \geq 0 .
$$

Applying Theorem 2.3 for a smooth function $u: S M \rightarrow \mathbb{R}$, we get

$$
\int_{S M}(\mathbf{F} V u)^{2} d \mu-\int_{S M} \mathbb{K}(V u)^{2} d \mu=\int_{S M}(V \mathbf{F} u)^{2} d \mu-\int_{S M}(\mathbf{F} u)^{2} d \mu,
$$

If $\mathbf{F} u=f$, then it is obvious that the right-hand side of (42) is nonpositive. Thus

$$
\int_{S M}(V \mathbf{F} u)^{2} d \mu-\int_{S M}(\mathbf{F} u)^{2} d \mu=-\int_{S M} f^{2} d \mu \leq 0 .
$$


Combining this with (41) written for $\varphi=V u$ yields $f=0$. The proof of Theorem 1.6 is complete.

\section{REFERENCES}

[1] Y. M. Assylbekov, N. S. Dairbekov, Hopf type rigidity for thermostats, Ergodic Theory and Dynamical Systems, 34, No.6 (2014) 1761-1769.

[2] W. Ballmann, K. Burns, M. Brin, On surfaces without conjugate points, J. Differential Geom. 25 (1987) 249-273.

[3] D. Bao, S.-S. Chern, Z. Shen, An introduction to Riemann-Finsler geometry, Graduate Texts in Mathematics, 200. Springer-Verlag, New York, 2000.

[4] N. S. Dairbekov, Integral geometry problem for nontrapping manifolds, Inverse Problems 22 (2006) 431-445.

[5] N. S. Dairbekov, G. P. Paternain, On the cohomological equation of magnetic flows, Matemática Contemporânea 34 (2008) 155-193.

[6] N. S. Dairbekov, G. P. Paternain, Entropy production in Gaussian thermostats, Comm. Math. Phys. 269 (2007) 533-543.

[7] N. S. Dairbekov, G. P. Paternain, Rigidity properties of Anosov optical hypersurfaces, Ergodic Theory and Dynamical Systems 28 (2008) 707-737.

[8] N. S. Dairbekov, G. P. Paternain, Longitudinal KAM-cocycles and action spectra of magnetic flows, Math. Res. Lett. 12 (2005) 719-730.

[9] N. S. Dairbekov, G. P. Paternain, P. Stefanov, G. Uhlmann, The boundary rigidity problem in the presence of a magnetic field, Advances in Mathematics 216, No. 2 (2007) 535-609.

[10] B. Frigyik, P. Stefanov, G. Uhlmann, The X-Ray Transform for a Generic Family of Curves and Weights, J. Geom. Anal. 18, No. 1 (2008) 81-97.

[11] V. Guillemin, D. Kazhdan, Some inverse spectral results for negatively curved 2maniifolds, Topology 19 (1980) 301-312.

[12] E. Ghys, Flots d'Anosov sur les 3-variétés fibrées en cercles, Ergodic Theory and Dynamical Systems 4 (1984) 67-80.

[13] S. Holman, P. Stefanov, The weighted Doppler transform, Inverse Problems and Imaging 4, No. 1 (2010) 111-130.

[14] E. Hopf, Closed surfaces without conjugate points, Proc. Nat. Acad. Sci. 34 (1948) 47-51.

[15] D. Jane, G. P. Paternain, On the injectivity of the X-ray transform for Anosov thermostats, Discrete and Continuous Dynamical Systems 24, No. 2 (2009) 471-488.

[16] R. de la Llave, J. M. Marco, R. Moriyon, Canonical pertubation theory of Anosov systems and regularity for the Livsic cohomology equation, Ann. Math. 123 (1986) $537-611$.

[17] R. G. Mukhometov, On the problem of integral geometry, Math. Problems of Geophysics. Akad. Nauk. SSSR, Sibirsk. Otdel., Vychisl. Tsentr, Novosibirsk, 6, No. 2 (1975) 212-242 (in Russian).

[18] R. G. Mukhometov, Inverse kinematic problem of seismic on the plane, Math. Problems of Geophysics. Akad. Nauk. SSSR, Sibirsk.Otdel., Vychisl. Tsentr, Novosibirsk, 6, No. 2 (1975) 243-252 (in Russian).

[19] G. P. Paternain, Geodesic flows, Progress in Mathematics, 180 Birghäuser 1999.

[20] G. P. Paternain, The longitudinal KAM-cocycle of a magnetic flow, Math. Proc. Cambridge Philos. Soc. 139 (2005) 307-316.

[21] S. Saks, Theory of the Integral, 2nd rev. ed., Hafner Publishing, New York, 1937. 
[22] V. A. Sharafutdinov, An integral geometry problem in a nonconvex domain, Siberian Math. J. 46, No. 6 (2002) 1159-1168.

[23] V. A. Sharafutdinov, Ray transform on Riemannian Manifolds. Eight Lectures on Integral Geometry, http://www.math.nsc.ru/ ${ }^{\sim}$ sharafutdinov/publ.html, 1999.

[24] M. Wojtkowski, Magnetic flows and Gaussian thermostats on manifolds of negative curvature, Fundamenta Math. 163 (2000) 177-191.

Department of Mathematics, University of Washington, Seattle, WA 981954350, USA

E-mail address: y_assylbekov@yahoo.com

Kazakh British Technical University, Tole Bi 59, 050000 Almaty, KazaKHSTAN

E-mail address: Nurlan.Dairbekov@gmail.com 